\title{
Microwave-Assisted Synthesis of Phenothiazine and Quinoline Derivatives
}

\author{
Luiza Găină $^{1}$, Castelia Cristea ${ }^{1}{ }^{*}$, Claudia Moldovan ${ }^{1}$, Dan Porumb ${ }^{1}$, Emanoil Surducan ${ }^{2}$ \\ Călin Deleanu ${ }^{3}$, Abdalah Mahamoud ${ }^{4}$, Jacques Barbe ${ }^{4}$ and Ioan A. Silberg ${ }^{1}$ \\ 1 "Babeş-Bolyai" University, Faculty of Chemistry and Chemical Engineering, Organic Chemistry \\ Department, Cluj-Napoca-Ro E-mail: gluiza@chem.ubbcluj.ro, E-mail: castelia@chem.ubbcluj.ro,E- \\ mail: pdan@chem.ubbcluj.ro \\ 2 INCDTIM-Cluj-Napoca-Ro E-mail: esurducan@gmail.com \\ 3 Institute of Organic Chemistry, National NMR Laboratory, Bucharest-Ro. E-mail: calind@ @rdslink.ro \\ 4 Universitée de la Mediteranée, Faculté de Pharmacie, Marseille CEDEX 5, E-mail: \\ barbe@pharmacie.univ-mrs.fr E-mail: mahamoud@pharmacie.univ-mrs.fr \\ * Author to whom correspondence should be addressed.
}

Received: 27 October 2006 / Accepted: 12 February 2007 / Published: 13 February 2007

\begin{abstract}
Application of a dynamic microwave power system in the chemical synthesis of some phenothiazine and quinoline derivatives is described. Heterocyclic ring formation, aromatic nucleophilic substitution and heterocyclic aldehydes/ketones condensation reactions were performed on solid support, or under solvent free reaction conditions. The microwave-assisted Duff formylation of phenothiazine was achieved. Comparison of microwave-assisted synthesis with the conventional synthetic methods demonstrates advantages related to shorter reaction times and in some cases better reaction yields.
\end{abstract}

Keywords: MAOS, Quinoline, Pyrido[3,2-g]quinoline, Phenothiazine, formylation.

\section{Introduction}

Microwave assisted organic synthesis became an increasingly popular technique in academic and industrial research laboratories, due to certain advantages, particularly shorter reaction times and rapid optimization of chemical reactions.

The experimental technique applied for the organic syntheses described below, is based on microwave power processing of materials using a dynamic control of the microwave power magnetron 
[1-3]. The aim of this work was to test the efficiency of this new dynamic microwave power system in the organic synthesis by optimizing the chemical synthesis of some interesting phenothiazine and quinoline derivatives.

Syntheses of symmetrically 4,6-bis-substituted amino-, oxo- and thio-pyrido[3,2-g]quinoline derivatives were previously reported [4,5] and their MDR reversal activity [6] was demonstrated. Unsymmetrical substituted derivatives were also synthesized with the aim to investigate their biological activity $[7,8]$. The cyclization reaction of substituted diphenylamines for phenothiazine ring formation was achieved by microwave-assisted synthesis, even in the presence of bulky substituents [9], which dramatically reduced the reaction yields when classical methods were employed. Arylsubstituted phenothiazinyl-enones and higher homologues (e.g. bis-chalcones) [10] are compounds, which could develop unconventional physical properties due to their structure combining the electron donor effects of the phenothiazine nucleus with those of an extended $\pi$ conjugated system.

\section{Results and Discussion}

The microwave assisted syntheses of quinoline and phenothiazine derivatives were performed in the resonance cavity of a dynamic microwave power system using microwave irradiation at variables duty cycles and power levels. The microwave input in material is controlled in every second from $0 \%$ to $100 \%$ of the time by a dynamic control of the microwave power magnetron. The reactions took place at normal pressure (in an open vessel). The processing techniques employed were: i) "dry media" procedure, ii) solvent heating and iii) simultaneous cooling method in the presence of a solvent. Several solid supports such as silica gel, alumina and clay (bentonite), were tested in dry media procedures.

The microwave assisted syntheses of quinoline derivatives are described by the chemical equations presented in scheme 1 . Table 1 presents a comparison between the results obtained by microwave assisted synthesis, versus conventional heating method [7,8]. The Skraup synthesis of 7-amino-8methylquinoline 1 [11,12] was performed starting with a mixture of 2,6-diaminotoluene, glycerol, sulfuric acid and arsenic pentoxide. Even though the reaction yield was not improved, an important decrease of the reaction time was achieved (table 1). The condensation reaction of 1 with the ethyl(etoxymethylene)-cyanoacetate on silica gel solid support gave a dramatic decrease of the reaction time simultaneous to an increase of the reaction yield.

The cyclization reaction of ethyl-3-(8-methylquinolin-7-ylamino)-2-cyanoacrylate 2 and the reaction of 3-cyano-10-methyl-pyrido[3,2-g]quinoline-4-one $\mathbf{3}$ with tetra-phosphorus decasulfide were performed on silica gel, or aluminosilicate clay (acidic bentonite). Variations of the reaction yields were observed according to the nature of the solid support employed. 4-Chloro-3-cyano-10-methylpyrido[3,2-g]quinoline 5 was obtained by the reaction of $\mathbf{3}$ with phosphorus oxychloride adsorbed on silica gel, in a significantly shorter reaction time. 
<smiles>CCOC/C=C(\CC)C(=O)OCC</smiles>
1

2

3<smiles>Cc1c2ncccc2cc2c(=S)c(C#N)c[nH]c12</smiles>

4

$\mathrm{RX}$ $\mathrm{K}_{2} \mathrm{CO}_{3}$ DMF

$\mid \begin{aligned} & \mathrm{RX} \\ & \mathrm{K}_{2} \mathrm{CO}_{3} \\ & \mathrm{DMF}\end{aligned}$

$\left\{\begin{array}{l}\mathrm{R}-\mathrm{NH}_{2} \\ \mathrm{~K}_{2} \mathrm{CO}_{3} \\ \mathrm{DMF}\end{array}\right.$<smiles>Cc1c2ncccc2cc2c(SP)c(C#N)cnc12</smiles>

6

6a $\mathrm{R}=-\mathrm{CH}_{2} \mathrm{CH}_{2} \mathrm{CH}_{2}-\mathrm{N}\left(\mathrm{CH}_{3}\right)_{2}$

6b $\mathrm{R}=-\mathrm{CH}_{2} \mathrm{CH}_{2} \mathrm{~N}$<smiles>[2H]c1c(C#N)cnc2c(C)c3ncccc3cc12</smiles>

7<smiles>[R]CCN1CCOCC1</smiles><smiles>[R]Nc1c(C#N)cnc2c(C)c3ncccc3cc12</smiles>

$\mathrm{R}=-\mathrm{CH}_{2} \mathrm{CH}_{2} \mathrm{CH}_{2}-\mathrm{N}\left(\mathrm{CH}_{3}\right)_{2}$

Scheme 1. Chemical reactions for the synthesis of quinoline derivatives.

Table 1. Preparation of quinoline derivatives using a dynamic microwave power system (50\%duty cycle, power level 400W).

\begin{tabular}{|c|c|c|c|c|c|c|c|}
\hline \multirow{2}{*}{ Compound } & \multicolumn{2}{|c|}{ Temp. $\left({ }^{\circ} \mathrm{C}\right)$} & \multicolumn{2}{c|}{ Time (min) } & \multicolumn{2}{c|}{ Yield (\%) } & \multirow{2}{*}{$\begin{array}{l}\text { Procedure } \\
\text { support }\end{array}$} \\
\cline { 2 - 7 } & $\Delta$ & MW & $\Delta$ & MW & $\Delta$ & MW & \\
\hline $\mathbf{1}$ & 140 & 132 & 270 & 33 & 47 & 32 & ii) $\mathrm{H}_{2} \mathrm{O}$ \\
\hline $\mathbf{2}$ & 110 & 99 & 120 & 1 & 68 & 95 & i) $\mathrm{SiO}_{2}$ \\
\hline $\mathbf{3}$ & 250 & 161 & 120 & 6 & 70 & 75 & i) $\mathrm{SiO}_{2}$ \\
& & 200 & & 6 & & 88 & i) bentonite \\
\hline $\mathbf{4}$ & 80 & 129 & 240 & 4 & 96 & 98 & i) $\mathrm{SiO}_{2}$ \\
& & 134 & & 2 & & 67 & i) bentonite \\
\hline $\mathbf{5}$ & 100 & 129 & 120 & 1 & 94 & 94 & i) $\mathrm{SiO}_{2}$ \\
\hline $\mathbf{6 a}$ & 80 & 141 & 240 & 2 & 33 & 60 & i) $\mathrm{SiO}_{2}$ \\
\hline $\mathbf{6 b}$ & 80 & 133 & 240 & 1 & 52 & 83 & i) $\mathrm{SiO}_{2}$ \\
\hline $\mathbf{7}$ & 120 & 125 & 240 & 4 & 29 & 88 & i) $\mathrm{SiO}_{2}$ \\
\hline $\mathbf{8}$ & 80 & 113 & 180 & 3 & 39 & 67 & i) $\mathrm{SiO}_{2}$ \\
\hline
\end{tabular}

A i) "dry media" procedure, ii) solvent heating and iii) simultaneous cooling method

The syntheses of the S-alkyl-3-cyano-10-methyl-pyrido[3,2-g]quinoline 6a,b, O-alkyl-3-cyano-10methyl-pyrido[3,2-g]quinoline 7 and $\mathrm{N}$-alkyl-3-cyano-10-methyl-pyrido[3,2-g]quinoline $\mathbf{8}$ gave the best results when silica gel solid support was employed, showing important improvements of the reactions yields. 
The microwave assisted syntheses of phenothiazine derivatives are described by the chemical equations presented in scheme 2. Table 2 presents a comparison between the results obtained by microwave assisted synthesis, versus conventional heating method [9].

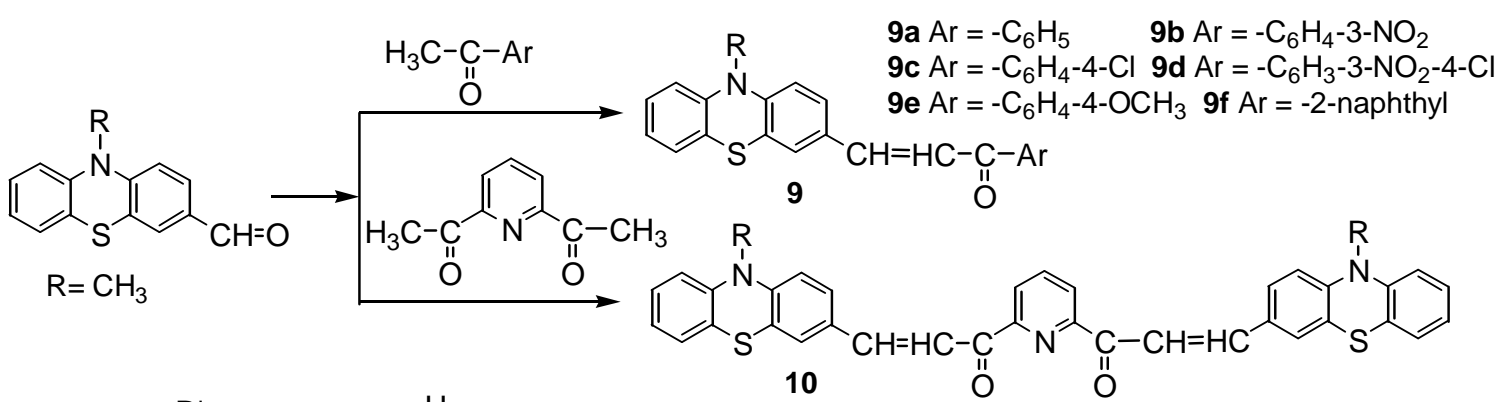<smiles>CCN1c2ccc(C(C)=O)cc2Sc2cc(C(C)=O)ccc21</smiles><smiles>CCN1c2ccc(C(=O)C=CI)cc2Sc2cc(C(=O)C=CC=[18O])ccc21</smiles><smiles>c1ccc2c(c1)Nc1ccccc1S2</smiles><smiles>CCCCCCCCC(C)=O</smiles><smiles>O=Cc1ccc2c(c1)Sc1ccccc1N2</smiles>

$11 \mathrm{Ar}=-\mathrm{C}_{6} \mathrm{H}_{4}-4-\mathrm{NO}_{2}$

Scheme 2. Chemical reactions for the synthesis of phenothiazine derivatives.

Table 2. Preparation of phenothiazine derivatives using a dynamic microwave power system (50\%duty cycle, power level 400W).

\begin{tabular}{|c|c|c|c|c|c|c|c|}
\hline \multirow[t]{2}{*}{ Compound } & \multicolumn{2}{|c|}{ Temp. $\left({ }^{\circ} \mathrm{C}\right)$} & \multicolumn{2}{|c|}{ Time (min) } & \multicolumn{2}{|c|}{ Yield (\%) } & \multirow{2}{*}{$\begin{array}{l}\text { Procedure } \\
\text { support }\end{array}$} \\
\hline & $\Delta$ & MW & $\Delta$ & MW & $\Delta$ & MW & \\
\hline $9 \mathbf{a}$ & 50 & $\begin{array}{l}68 \\
68 \\
\end{array}$ & 360 & $\begin{array}{l}9 \\
15\end{array}$ & 62 & $\begin{array}{l}80 \\
70\end{array}$ & $\begin{array}{l}\text { i) } \mathrm{SiO}_{2} \\
\text { iii) } \mathrm{EtOH}\end{array}$ \\
\hline $9 b$ & 50 & $\begin{array}{l}96 \\
78\end{array}$ & 240 & $\begin{array}{l}5 \\
10\end{array}$ & 70 & $\begin{array}{l}70 \\
80\end{array}$ & $\begin{array}{l}\text { i) } \mathrm{SiO}_{2} \\
\text { iii) } \mathrm{EtOH}\end{array}$ \\
\hline $9 c$ & 50 & $\begin{array}{l}82 \\
60 \\
\end{array}$ & 240 & $\begin{array}{l}6 \\
6 \\
\end{array}$ & 85 & $\begin{array}{l}65 \\
75 \\
\end{array}$ & $\begin{array}{l}\text { i) } \mathrm{SiO}_{2} \\
\text { iii) } \mathrm{EtOH}\end{array}$ \\
\hline $9 d$ & 50 & $\begin{array}{l}68 \\
60 \\
\end{array}$ & 240 & $\begin{array}{l}15 \\
10 \\
\end{array}$ & 60 & $\begin{array}{l}75 \\
88\end{array}$ & $\begin{array}{l}\text { i) } \mathrm{SiO}_{2} \\
\text { iii) } \mathrm{EtOH}\end{array}$ \\
\hline $9 e$ & 50 & $\begin{array}{l}65 \\
70 \\
\end{array}$ & 720 & $\begin{array}{l}12 \\
4 \\
\end{array}$ & 56 & $\begin{array}{l}75 \\
80 \\
\end{array}$ & $\begin{array}{l}\text { i) } \mathrm{SiO}_{2} \\
\text { ii) } \mathrm{EtOH}\end{array}$ \\
\hline $9 f$ & 50 & $\begin{array}{l}74 \\
72\end{array}$ & 720 & $\begin{array}{l}12 \\
4\end{array}$ & 60 & $\begin{array}{l}85 \\
90\end{array}$ & $\begin{array}{l}\text { i) } \mathrm{SiO}_{2} \\
\text { ii) } \mathrm{EtOH}\end{array}$ \\
\hline 10 & 50 & $\begin{array}{l}85 \\
70 \\
\end{array}$ & 480 & $\begin{array}{l}9 \\
9 \\
\end{array}$ & 50 & $\begin{array}{l}50 \\
70 \\
\end{array}$ & $\begin{array}{l}\text { i) } \mathrm{SiO}_{2} \\
\text { iii) } \mathrm{EtOH}\end{array}$ \\
\hline 11 & 50 & $\begin{array}{l}80 \\
77 \\
\end{array}$ & 300 & $\begin{array}{l}7 \\
3 \\
\end{array}$ & 40 & $\begin{array}{l}65 \\
80 \\
\end{array}$ & $\begin{array}{l}\text { i) } \mathrm{SiO}_{2} \\
\text { iii) } \mathrm{EtOH}\end{array}$ \\
\hline 12 & 120 & 94 & 1060 & 30 & 40 & 50 & iii) $\mathrm{EtOH}$ \\
\hline
\end{tabular}

A i) "dry media" procedure, ii) solvent heating, iii) simultaneous cooling method.

The heterocyclic chalcones 9a-f were obtained by the condensation of 3-formyl-10methylphenothiazine with substituted acetophenones. Good reaction yields were obtained under microwave irradiation, using both dry media procedure and simultaneous cooling method. For the 
preparation of heterocyclic bis-chalcones $\mathbf{1 0}$ and $\mathbf{1 1}$ the simultaneous cooling method is recommended by higher reaction yields, even though dry media procedure give also satisfactory results.

Duff formylation reaction was applied for the first time to a phenothiazine substrate. The microwave-assisted Duff formylation of phenothiazine with urotropine in acetic acid gave unexpectedly good yields of 10(H)-3-formyl-phenothiazine 12, in significantly shorter reaction time as compared to classical reaction protocol.

Conclusions: The dynamic microwave power system employed offered an efficient heating of the materials; thus, reduced chemical reactions times and increased reaction yields were observed in most of the experiments performed.

\section{Experimental Section}

The reactions were performed in the resonance cavity of a dynamic microwave power system, designed at INCDTIM Cluj-Napoca, Romania.

Reagents from Merck and Aldrich Chemical Co. were used. Anhydrous Silica gel 60 (0.063-0.2 mm) was used as solid support after dehydration under microwave irradiation for 4 minutes.

TLC was used to monitor the reaction progress (Merck silica gel F 254 plates).

The structures of the reaction products were assigned according to NMR spectra recorded using a 400 $\mathrm{MHz}$ Brucker NMR spectrometer.

\subsection{General procedures}

i) Dry media procedure

The reagents were solved in a low boiling point solvent at room temperature; anhydrous microwave transparent inorganic solid support (silica gel, alumina or clay) was added and the solvent was removed under vacuum. The adsorbed reaction mixture was introduced in an open quartz tube, which was then subjected to microwave irradiation in the resonance cavity of the microwave power system. Initial and final sample temperatures were measured. The sample was cooled in an ice bath, and the irradiation was repeated several times. TLC was used to monitor the reaction progress. The reaction product was extracted with solvent; the extract was filtered, dried over anhydrous sodium sulfate and then the solvent was removed. The products were purified by recrystallization or column chromatography.

\section{ii) Solvent heating}

The reaction mixture solved in the properly choose solvent was introduced in an open quartz tube, which was then subjected to microwave irradiation. Initial and final sample temperature was measured. The sample was cooled in an ice bath, and the irradiation was repeated several times. TLC was used to monitor the reaction progress. The solvent was removed under vacuum and the product was purified by recrystallization or column chromatography.

\section{iii ) Simultaneous Cooling Method}

The reaction mixture solved in the properly choose solvent was subjected to microwave irradiation in a reaction vessel provided with a cooling mantle. During irradiation, the circulation of the cooling agent 
ensures the control of the reaction temperature and avoids the super heating of the solvent. Work-up of the reaction product was similar to the methods indicated above.

\section{7-Amino-8-methyl-quinoline 1}

A mixture of $4 \mathrm{mmol}$ 2,6-diaminotoluene $(0.5 \mathrm{~g})$, glycerol $(2.5 \mathrm{~mL})$, arsenic $(\mathrm{V})$ oxide $(2.1 \mathrm{~g})$ and sulfuric acid (conc. $4.2 \mathrm{~mL}$ ) was subjected to microwave irradiation according to procedure ii). After cooling at room temperature, the reaction mixture was poured into an ice-water mixture $(15 \mathrm{~mL})$ and alkalinized at $\mathrm{pH}=9-10$. The precipitate obtained was filtered, washed with cold water and crystallized from water; $0.2 \mathrm{~g}$ of yellow powder (m.p. $=128^{\circ} \mathrm{C}$ ) was obtained.

NMR (400MHz, $\left.\mathrm{CHCl}_{3}-\mathrm{d}_{1}\right): \boldsymbol{\delta}_{\mathbf{H}}(\mathrm{ppm}) 2.59$ (s, 3H), $4.0\left(\mathrm{~s},-\mathrm{NH}_{2}\right), 7.0$ (d, 1H, J=8.8 Hz), 7.14 (dd, 1H, $\mathrm{J}=4.4 \mathrm{~Hz}, \mathrm{~J}=8.2 \mathrm{~Hz}), 7.49$ (d, 1H, J = $8.8 \mathrm{~Hz}$ ), 7.9 (dd, 1H, J=2 Hz, J=8.2 Hz), 8.8 (dd, 1H, J=2 Hz, $\mathrm{J}=4.4 \mathrm{~Hz}$ ). $\boldsymbol{\delta}_{\mathbf{C}}$ (ppm) 10.25; 115.03; 117.32; 118.38; 122.56; 126.17; 136.06; 144.79; 148.26; 149.61 .

\section{(8-Methyl-quinoline-7-aminomethylene)ethyl cyanoacetate 2}

A mixture of $3.1 \mathrm{mmol} 7$-amino-8-methyl-quinoline $(0.5 \mathrm{~g}$,) and $9.3 \mathrm{mmol}$ ethyl-(etoxymethylene)cyanoacetate $(1.5 \mathrm{~g}$, ) was subjected to microwave irradiation. The reaction product $(0.8 \mathrm{~g}$ of white powder, m.p. $=206{ }^{\circ} \mathrm{C}$ ) was obtained according to procedure i) using $10 \mathrm{~g}$ silica gel solid support. Crystallization from ethanol gave the pure product.

NMR (400MHz, DMSO-d $\left.)_{6}\right) \boldsymbol{\delta}_{\mathbf{H}}(\mathrm{ppm}): 1.24\left(\mathrm{t}, 3 \mathrm{H}, \mathrm{J}=7 \mathrm{~Hz},-\mathrm{CH}_{3}\right), 2.84\left(\mathrm{~s}, 3 \mathrm{H},-\mathrm{CH}_{3}\right), 4.35(\mathrm{q}, 2 \mathrm{H}$, $\mathrm{J}=7), 7.39(\mathrm{dd}, 1 \mathrm{H}, \mathrm{J}=4 \mathrm{~Hz}, \mathrm{~J}=8.2 \mathrm{~Hz}), 7.42$ (d, 1H, J=8.8Hz), $7.77(\mathrm{~d}, 1 \mathrm{H}, \mathrm{J}=8.8 \mathrm{~Hz}), 8.01$ (d, 1H, $\mathrm{J}=13 \mathrm{~Hz}), 8.12$ (dd, 1H, J=1.6 Hz, J=8.2 Hz), 8.96 (dd, 1H, J=1.6 Hz, J=4 Hz), 11,31 (d, 1H, J=13 Hz, $\mathrm{NH}) . \boldsymbol{\delta}_{\mathbf{C}}(\mathrm{ppm}): 10.92 ; 14.33 ; 61.44 ; 76.3(\mathrm{CN}) ; 115,00 ; 117.81 ; 120.60 ; 124.43 ; 125.97 ; 127.51$; $136.33 ; 136.43 ; 147.54,150.68 ; 151.81 ; 167.74$.

\section{0-methyl-4-oxo-pyrido[3,2-g]quinolin-3-carbonitrile 3}

$1.7 \mathrm{mmol}$ (8-methyl-quinoline-7-aminomethylene)-ethylcyanoacetate $(0.5 \mathrm{~g}$,$) were subjected to$ microwave irradiation according to procedure $\mathrm{i}$ ), using $10 \mathrm{~g}$ silica gel solid support and $10 \mathrm{~g}$ clay (acidic bentonite) respectively. The reaction product $\left(0.3 \mathrm{~g} / 0.35 \mathrm{~g}\right.$ of white powder, m.p. $\left.=292{ }^{\circ} \mathrm{C}\right)$ was obtained by pouring the dimethylformamide extract in water, followed by filtration of the precipitate.

NMR (400MHz, DMSO-d $\left.)_{6}\right): \boldsymbol{\delta}_{\mathbf{H}}(\mathrm{ppm}): 2.93$ (s, 1H), 7.57 (dd, 1H, J=4 Hz, J=8.4 Hz), 8.59 (dd, 1H, $\mathrm{J}=1.6 \mathrm{~Hz}, \mathrm{~J}=8.4 \mathrm{~Hz}$ ), 8.65 (s, 1H), 8.71 (s, 1H), 9.06 (dd, 1H, J=1.6 Hz, J=4 Hz). $\boldsymbol{\delta}_{\mathrm{C}}$ (ppm): 11.19; 91.41 (-CN); 116.91; 121.56; 123.94; 124.52; 124.96; 125.01; 136.26; 138.43; 146.49; 148.51; 153.18; 175.77 .

\section{0-methyl-4-thioxo-pyrido[3,2-g]quinolin-3-carbonitrile 4}

A mixture of $2.5 \mathrm{mmol} 3\left(0.6 \mathrm{~g}\right.$, ) and $1.1 \mathrm{mmol}$ tetra-phosphorus decasulfide $\mathrm{P}_{4} \mathrm{~S}_{10}(0.5 \mathrm{~g}$, ) was subjected to microwave irradiation according to procedure $\mathrm{i}$ ), using $1 \mathrm{~g}$ silica gel solid support and $1 \mathrm{~g}$ clay (acidic bentonite) respectively. The reaction product $\left(0.6 \mathrm{~g} / 0.4 \mathrm{~g}\right.$ of orange powder, m.p. $=196{ }^{\circ} \mathrm{C}$ with decompn.) was obtained by pouring the dimethylformamide extract in water, followed by filtration of the precipitate. 
NMR (400MHz, DMSO-d 6 ): $\boldsymbol{\delta}_{\mathbf{H}}(\mathrm{ppm}): 2.99$ (s, 3H), 7.6 (dd, 1H, J=6.4 Hz, J=7.2 Hz), 8.60 (s, 1H), $8.64(\mathrm{dd}, 1 \mathrm{H}, \mathrm{J}=7.2 \mathrm{~Hz}, \mathrm{~J}=1.8 \mathrm{~Hz}), 9.10(\mathrm{dd}, 1 \mathrm{H}, \mathrm{J}=6.4 \mathrm{~Hz}, \mathrm{~J}=1.8 \mathrm{~Hz}), 9.11(\mathrm{~s}, 1 \mathrm{H}) . \boldsymbol{\delta}_{\mathrm{C}}(\mathrm{ppm}): 11.2$; $106.1 ; 114.3 ; 115.9 ; 116.2 ; 120.6 ; 126.8 ; 131.4 ; 134.9 ; 147.2 ; 148.8 ; 156.4 ; 159.8 ; 228.6$.

\section{4-Chloro-10-methyl-pyrido[3,2-g]quinolin-3-carbonitrile 5}

A mixture of $2.1 \mathrm{mmol} 3(0.5 \mathrm{~g})$ and phosphorous oxychloride $(0.2 \mathrm{~mL}$,) was subjected to microwave irradiation according to procedure $\mathrm{i}$ ), using $1 \mathrm{~g}$ silica gel solid support. $0.5 \mathrm{~g}$ of green-yellow powder, m.p. $=264{ }^{\circ} \mathrm{C}$ (with decompn.) were obtained.

NMR (400MHz, DMSO-d $\left.)_{6}\right): \boldsymbol{\delta}_{\mathbf{H}}(\mathrm{ppm}): 3.02$ (s, 3H), 7.38 (dd, 1H, J=8.4 Hz, J=4Hz), 8.34 (s, 1H), $8.44(\mathrm{dd}, 1 \mathrm{H}, \mathrm{J}=8.4 \mathrm{~Hz}, \mathrm{~J}=1,2 \mathrm{~Hz}), 8.60(\mathrm{~s}, 1 \mathrm{H}), 8.93$ (dd, 1H, J=4Hz, J=1.2 Hz). $\boldsymbol{\delta}_{\mathbf{C}}(\mathrm{ppm}): 12.2$; $104.3 ; 116.4 ; 120.8 ; 128.3 ; 128.4 ; 126.5 ; 135.2 ; 136.7 ; 137.5 ; 145.4 ; 149.5 ; 149.9 ; 154.8$.

\section{4-(3-N,N-dimethylamino-propylthio)-10-methyl-pyrido[3,2-g]quinolin-3-carbonitrile 6a}

A mixture of $1.4 \mathrm{mmol} 4(0.5 \mathrm{~g}), 5 \mathrm{mmol} \mathrm{N}, \mathrm{N}$-dimethylaminopropyl chloride hydrochloride $(0.8 \mathrm{~g})$ and anhydrous $\mathrm{K}_{2} \mathrm{CO}_{3}(0.6 \mathrm{~g})$, was subjected to microwave irradiation according to procedure $\mathrm{i}$ ), using $1 \mathrm{~g}$ silica gel solid support. $0.4 \mathrm{~g}$ of yellow powder, m.p. $=74{ }^{\circ} \mathrm{C}$ were obtained.

NMR (400MHz, $\left.\mathrm{CHCl}_{3}-\mathrm{d}_{1}\right): \boldsymbol{\delta}_{\mathbf{H}}(\mathrm{ppm}): 1.86(\mathrm{~m}, 2 \mathrm{H}), 2.19$ (s, 6H), $2.44(\mathrm{t}, 2 \mathrm{H}, \mathrm{J}=7.2 \mathrm{~Hz}), 3.37$ (s, 3H), 3.43 (t, 2H, J =7.2 Hz), 7.53 (dd, 1H, J=8.4 Hz, J=3.6 Hz), 8.98 (s, 1H), 8.41 (dd, 1H, J=8.4 Hz, J=1,2 Hz), 9.0 (s, 1H), $9.16(\mathrm{dd}, 1 \mathrm{H}, \mathrm{J}=3.6 \mathrm{~Hz}, \mathrm{~J}=1.6 \mathrm{~Hz}) . \boldsymbol{\delta}_{\mathrm{C}}(\mathrm{ppm}): 12.47 ; 27.88 ; 34.25$, 45.33; 57.75; $109.90 ; 117.25,121.98 ; 124.24 ; 124.58 ; 127.35 ; 137.46 ; 146.44 ; 149.61,138.21 ; 144.10 ; 152.55$; 153.2 .

\section{4-(morpholino-ethylthio)-10-methyl-pyrido[3,2-g]quinolin-3-carbonitrile 6b}

A mixture of $1.4 \mathrm{mmol} 4(0.5 \mathrm{~g}),, 4 \mathrm{mmol}$ 2-chloroethyl morpholine hydrochloride $(0.9 \mathrm{~g})$ and anhydrous $\mathrm{K}_{2} \mathrm{CO}_{3}(0.6 \mathrm{~g})$, was subjected to microwave irradiation according to procedure $\mathrm{i}$ ), using $1 \mathrm{~g}$ silica gel solid support. $0.6 \mathrm{~g}$ of yellow powder, m.p. $=159{ }^{\circ} \mathrm{C}$ were obtained.

NMR (400MHz, $\left.\mathrm{CHCl}_{3}-\mathrm{d}_{1}\right): \boldsymbol{\delta}_{\mathbf{H}}(\mathrm{ppm}): 2.39$ (t, 4H), 2.72 (t, 2H. J=6.4 Hz), 3.37 (s, 3H), 3.48 (s, 4H), $3.50(\mathrm{t}, 2 \mathrm{H}, \mathrm{J}=5.2 \mathrm{~Hz}), 7.53(\mathrm{dd}, 1 \mathrm{H}, \mathrm{J}=8.4 \mathrm{~Hz}, \mathrm{~J}=3.6 \mathrm{~Hz}), 8.97(\mathrm{~s}, 1 \mathrm{H}), 8.40(\mathrm{dd}, 1 \mathrm{H}, \mathrm{J}=8.4 \mathrm{~Hz}, \mathrm{~J}=1,2$ $\mathrm{Hz}), 9.03$ (s, 1H), $9.16(\mathrm{dd}, 1 \mathrm{H}, \mathrm{J}=3.6 \mathrm{~Hz}, \mathrm{~J}=1.6 \mathrm{~Hz}) . \boldsymbol{\delta}_{\mathrm{C}}(\mathrm{ppm}): 12.47\left(-\mathrm{CH}_{3}\right) ; 33.56 ; 58.29 ; 53.24$; $66.68 ; 110.02(-\mathrm{CN}) ; 152.57 ; 117.30 ; 137.40 ; 122.0 ; 124.31 ; 127.28 ; 153.90 ; 125.73 ; 146.39 ; 148.83$, $138.16 ; 144.06$;

\section{4-(morpholino-ethyloxy)-10-methyl-pyrido[3,2-g]quinolin-3-carbonitrile 7}

A mixture of $2.1 \mathrm{mmol} 3(0.5 \mathrm{~g}),, 2.1 \mathrm{mmol}$ 2-chloroethyl morpholine hydrochloride $(0.4 \mathrm{~g}$,) and anhydrous $\mathrm{K}_{2} \mathrm{CO}_{3}(0.6 \mathrm{~g})$, was subjected to microwave irradiation according to procedure $\mathrm{i}$ ), using $1 \mathrm{~g}$ silica gel solid support. $0.6 \mathrm{~g}$ of yellow powder, m.p. $=173{ }^{\circ} \mathrm{C}$ were obtained.

NMR (400MHz, $\left.\mathrm{CHCl}_{3}-\mathrm{d}_{1}\right): \boldsymbol{\delta}_{\mathbf{H}}(\mathrm{ppm}): 2.32(\mathrm{t}, 4 \mathrm{H}), 2.51(\mathrm{t}, 2 \mathrm{H}), 3.10(\mathrm{~s}, 3 \mathrm{H}), 3.54(\mathrm{t}, 4 \mathrm{H}), 4.50(\mathrm{t}$, 2H), 7.52 (dd, 1H, J=8.4 Hz, J=4.2 Hz), 8.25 (s, 1H), 8.37 (dd, 1H, J=8.4 Hz, J=1,8 Hz), 8.86 (s, 1H), $9.09(\mathrm{dd}, 1 \mathrm{H}, \mathrm{J}=3.9 \mathrm{~Hz}, \mathrm{~J}=2.1 \mathrm{~Hz}), \boldsymbol{\delta}_{\mathbf{C}}(\mathrm{ppm}) 12.6 ; 53.2 ; 54.2 ; 66.6 ; 66.4 ; 112.2 ; 117.30 ; 122.0$; $124.31 ; 125.73 ; 127.28 ; 137.40 ; 138.16 ; 144.06 ; 146.39 ; 148.83,152.57 ; 153.90$. 


\section{4-(1-propylamino)-10-methyl-pyrido[3,2-g]quinolin-3-carbonitrile 8}

A mixture of $1.9 \mathrm{mmol} 5(0.5 \mathrm{~g}),, 3.5 \mathrm{mmol} \mathrm{N}, \mathrm{N}$-dimethyl-1,3-propanediamine hydrochloride $(0.5 \mathrm{~g})$ and anhydrous $\mathrm{K}_{2} \mathrm{CO}_{3}(0.6 \mathrm{~g})$, was subjected to microwave irradiation according to procedure $\mathrm{i}$ ), using $1 \mathrm{~g}$ silica gel solid support. $0.4 \mathrm{~g}$ of yellow powder, m.p. $=158{ }^{\circ} \mathrm{C}$ were obtained.

NMR (400MHz, $\left.\mathrm{CHCl}_{3}-\mathrm{d}_{1}\right): \boldsymbol{\delta}_{\mathbf{H}}(\mathrm{ppm}): 2.42(\mathrm{t}, 3 \mathrm{H}), 2.77(\mathrm{~m}, 2 \mathrm{H}), 3.29(\mathrm{~s}, 3 \mathrm{H}), 4.04(\mathrm{t}, 2 \mathrm{H}), 4.05(\mathrm{t}$, 1H), 7.44 (dd, 1H, J=5.4 Hz, J=0.9 Hz), 8.16 (s, 1H), 8.32 (dd, 1H, J=8.1 Hz, J=6,6 Hz), 8.67 (s, 1H), $9.10(\mathrm{dd}, 1 \mathrm{H}, \mathrm{J}=2.7 \mathrm{~Hz} ; \mathrm{J}=1.8 \mathrm{~Hz}) . \boldsymbol{\delta}_{\mathbf{C}}(\mathrm{ppm}): 12.6 ; 26.6 ; 44.2 ; 42.8 ; 56.4 ; 91.41 ; 116.91 ; 121.56$; $123.94 ; 124.52 ; 124.96 ; 125.01 ; 136.26 ; 138.43 ; 146.49 ; 148.51 ; 153.18 ; 155.77$.

\section{(E)-1-(10-methyl-10Hphenothiazin-3-yl)-3-phenyl-propenone 9a}

A mixture of $0.3 \mathrm{mmol} 10$-methyl-3-formylphenothiazine $(0.073 \mathrm{~g}), 0.3 \mathrm{mmol}$ acetophenone $(0.036 \mathrm{~g})$ and $\mathrm{NaOH}$ catalyst was subjected to microwave irradiation. The reaction product (yellow powder, m.p. $=147^{\circ} \mathrm{C}$ ) was obtained according to procedure i) $0.08 \mathrm{~g}$ reaction product using $1 \mathrm{~g}$ silica gel solid support, or according to procedure iii) $0.07 \mathrm{~g}$ reaction product using $50 \mathrm{~mL}$ ethanol solvent.

NMR (400MHz, $\left.\mathrm{CHCl}_{3}-\mathrm{d}_{1}\right): \boldsymbol{\delta}_{\mathbf{H}}(\mathrm{ppm}): 3.41(\mathrm{~s}, 3 \mathrm{H}), 6.80$ (d, 1H J=8.Hz), 6.83 (d, 1H J=8.5Hz), 6.96 $(\mathrm{t}, 1 \mathrm{H}, \mathrm{J}=7.6 \mathrm{~Hz}, \mathrm{~J}=7.4 \mathrm{~Hz}), 7.14(\mathrm{dd}, 1 \mathrm{H}, \mathrm{J}=7.4 \mathrm{~Hz}, \mathrm{~J}=1.4 \mathrm{~Hz}), 7.18(\mathrm{~m}, 1 \mathrm{H}), 7.40(\mathrm{~d}, 1 \mathrm{H}, \mathrm{J}=15.6 \mathrm{~Hz})$, $7.42(\mathrm{dd}, 2 \mathrm{H}, \mathrm{J}=8 \mathrm{~Hz}, \mathrm{~J}=1.8 \mathrm{~Hz}), 7.50(\mathrm{t}, 2 \mathrm{H}, \mathrm{J}=7.4 \mathrm{~Hz}, \mathrm{~J}=7.8 \mathrm{~Hz}), 7.58(\mathrm{t}, 1 \mathrm{H}), 7.71(\mathrm{~d}, 1 \mathrm{H}, \mathrm{J}=15.6 \mathrm{~Hz})$, $8.00(\mathrm{dd}, 2 \mathrm{H} \mathrm{J}=7.8 \mathrm{~Hz}, \mathrm{~J}=1.2 \mathrm{~Hz}) . \boldsymbol{\delta}_{\mathbf{C}}(\mathrm{ppm}): 35.57,114.09,114.44,119.92,122.61,123.99,126.28$, 127.27, 127.68, 128.44, 128.60, 129.11, 129.33, 132.63, 138.45, 143.80, 144.75, 147.86, 190.36 .

\section{(E)-1-(10-methyl-10H phenothiazin-3-yl)-3-(3-nitrophenyl)-propenone 9b}

A mixture of $0.15 \mathrm{mmol} 10$-methyl-3-formylphenothiazine $(0.037 \mathrm{~g}), 0.15 \mathrm{mmol}$ 3-nitro-acetophenone $(0.026 \mathrm{~g})$ and $\mathrm{NaOH}$ catalyst was subjected to microwave irradiation. The reaction product (orange powder, m.p. $180{ }^{\circ} \mathrm{C}$ ) was obtained according to procedure i) $0.04 \mathrm{~g}$ reaction product using $1 \mathrm{~g}$ silica gel solid support, or according to procedure iii) $0.05 \mathrm{~g}$ reaction product using $50 \mathrm{~mL}$ ethanol solvent.

NMR (400MHz, $\left.\mathrm{CHCl}_{3}-\mathrm{d}_{1}\right): \boldsymbol{\delta}_{\mathbf{H}}(\mathrm{ppm}): 3.42$ (s, 3H); 6.84, (m. 2H), 6.97, (m, 1H), 7.14 (dd, 1H), 7.19 $(\mathrm{m}, 1 \mathrm{H}), 7.39,(\mathrm{~d}, 1 \mathrm{H}), 7.44(\mathrm{~m}, 2 \mathrm{H}), 7.70,(\mathrm{t}, 1 \mathrm{H}), 7.79(\mathrm{~d}, 1 \mathrm{H}), 8.34(\mathrm{~m}, 1 \mathrm{H}), 8.42,(\mathrm{~m}, 1 \mathrm{H}) ; 8.82$, (t, $1 \mathrm{H}) . \boldsymbol{\delta}_{\mathbf{C}}$ (ppm): $35.63,114.13,114.54,118.27,122.48,123.19,123.27,124.14,126.57,126.89,127.29$, $127.74,128.72,129.55,129.85,134.05,139.81,144.53,145.72,148.40,148.44,187.69$.

\section{(E)-1-(10-methyl-10H phenothiazin-3-yl)-3-(3-nitrophenyl)-propenone 9c}

A mixture of $0.15 \mathrm{mmol}$ 10-methyl-3-formylphenothiazine $(0.037 \mathrm{~g}), \quad 0.15 \mathrm{mmol} \quad 4-$ chloroacetophenone $(0.025 \mathrm{~g})$ and $\mathrm{NaOH}$ catalyst was subjected to microwave irradiation. The reaction product (orange powder, m.p. $153{ }^{\circ} \mathrm{C}$ ) was obtained according to procedure i) $0.04 \mathrm{~g}$ reaction product using $1 \mathrm{~g}$ silica gel solid support, or according to procedure iii) $0.045 \mathrm{~g}$ reaction product using $50 \mathrm{~mL}$ ethanol solvent.

NMR (400MHz, $\left.\mathrm{CHCl}_{3}-\mathrm{d}_{1}\right): \boldsymbol{\delta}_{\mathbf{H}}(\mathrm{ppm}): 3.32(\mathrm{~s}, 3 \mathrm{H}), 6.73(\mathrm{~d}, 1 \mathrm{H}), 6.76(\mathrm{~d}, 1 \mathrm{H}), 6.90(\mathrm{~m}, 1 \mathrm{H}), 7.07,(\mathrm{~d}$, $1 \mathrm{H}), 7.11,(\mathrm{~m}, 1 \mathrm{H}), 7.27,(\mathrm{~d}, 1 \mathrm{H}), 7.35,(\mathrm{~m}, 2 \mathrm{H}), 7.39(\mathrm{~d}, 2 \mathrm{H}), 7.64,(\mathrm{~d}, 1 \mathrm{H}), 7.88(\mathrm{~d}, 2 \mathrm{H}) . \boldsymbol{\delta}_{\mathrm{C}}(\mathrm{ppm})$ $35.66 ; 114.44 ; 114.09 ; 119.92 ; 122.61 ; 123.99,128.44 ; 126.28 ; 127.27 ; 127.68 ; 129.33,129.11$, $131.32 ; 132.63 ; 138.45,143.80,140.24 ; 144.75 ; 147.86 ; 189.62$. 


\section{(E)-1-(10-methyl-10H phenothiazin-3-yl)-3-(4-chloro-3-nitrophenyl)-propenone 9d}

A mixture of $0.15 \mathrm{mmol}$ 10-methyl-3-formylphenothiazine $(0.037 \mathrm{~g}), 0.15 \mathrm{mmol}$ 4-chloro-3nitroacetophenone $(0.03 \mathrm{~g})$ and $\mathrm{NaOH}$ catalyst was subjected to microwave irradiation. The reaction product (dark red powder, m.p. $173{ }^{\circ} \mathrm{C}$ with decomposition) was obtained according to procedure i) $0.05 \mathrm{~g}$ reaction product using $1 \mathrm{~g}$ silica gel solid support, or according to procedure iii) $0.06 \mathrm{~g}$ reaction product using $50 \mathrm{~mL}$ ethanol solvent.

NMR (400MHz, $\left.\mathrm{CHCl}_{3}-\mathrm{d}_{1}\right): \boldsymbol{\delta}_{\mathbf{H}}(\mathrm{ppm}): 3.42(\mathrm{~s}, 3 \mathrm{H}), 6.79(\mathrm{t}, 1 \mathrm{H}), 6.81(\mathrm{~d}, 1 \mathrm{H}), 6.84(\mathrm{~d}, 1 \mathrm{H}), 7.14(\mathrm{dd}$, $1 \mathrm{H}), 7.19(\mathrm{~m}, 1 \mathrm{H}), 7.31,(\mathrm{~d}, 1 \mathrm{H}), 7.44(\mathrm{~m}, 2 \mathrm{H}), 7.69(\mathrm{~d}, 1 \mathrm{H}), 7.78(\mathrm{~d}, 1 \mathrm{H}), 8.14(\mathrm{dd}, 1 \mathrm{H}), 8.48(\mathrm{~d}, 1 \mathrm{H})$. $\boldsymbol{\delta}_{\mathbf{C}}(\mathrm{ppm}) 35.63,114.12,114.55,117.66,121.4 ; 123.30,125.30,126.59,126.24 ; 127.28,127.75$, $129.66,130.32 ; 132.38,134.76 ; 136.98 ; 137.44 ; 144.26 ; 145.12 ; 146.06 ; 149.34 ; 189.62$.

\section{(E)-1-(10-methyl-10H phenothiazin-3-yl)-3-(4-methoxyphenyl)-propenone 9e}

A mixture of $0.15 \mathrm{mmol}$ 10-methyl-3-formylphenothiazine $(0.037 \mathrm{~g}), \quad 0.18 \mathrm{mmol} \quad 4$ methoxyacetophenone $(0.028 \mathrm{~g})$ and $\mathrm{NaOH}$ catalyst was subjected to microwave irradiation. The reaction product (yellow powder, m.p. $197{ }^{\circ} \mathrm{C}$ ) was obtained according to procedure i) $0.044 \mathrm{~g}$ reaction product using $1 \mathrm{~g}$ silica gel solid support, or according to procedure ii) $0.047 \mathrm{~g}$ reaction product using $50 \mathrm{~mL}$ ethanol solvent.

NMR (400MHz, $\left.\mathrm{CHCl}_{3}-\mathrm{d}_{1}\right): \boldsymbol{\delta}_{\mathbf{H}}(\mathrm{ppm}): 3.40(\mathrm{~s} ; 3 \mathrm{H}), 3.89(\mathrm{~s} ; 3 \mathrm{H}), 6.80(\mathrm{~d}, 1 \mathrm{H}), 6.83(\mathrm{dd} ; 1 \mathrm{H}), 6.96(\mathrm{~m}$, $1 \mathrm{H}) 6.98(\mathrm{~d}, 2 \mathrm{H}), 7.14(\mathrm{dd} ; 1 \mathrm{H}), 7.18(\mathrm{~m}, 1 \mathrm{H}), 7.40(\mathrm{dd}, 1 \mathrm{H}), 7.41(\mathrm{~d} ; 1 \mathrm{H}) 7.42(\mathrm{~d}, 1 \mathrm{H}), 7.70(\mathrm{~d}, 1 \mathrm{H})$, $8.03(\mathrm{~d} ; 1 \mathrm{H}) . \boldsymbol{\delta}_{\mathrm{C}}(\mathrm{ppm}): 35.5 ; 55.5 ; 113.8 ; 114.1 ; 114.4 ; 119.7 ; 122.6 ; 123.0 ; 123.9 ; 126.2 ; 127.2 ;$ $127.7 ; 129.0 ; 129.5 ; 130.7 ; 131.3 ; 142.9 ; 144.8 ; 147.6 ; 163.3 ; 188.5$.

\section{(E)-1-(10-methyl-10H phenothiazin-3-yl)-3-(2-naphthyl)-propenone 9f}

A mixture of $0.15 \mathrm{mmol} 10$-methyl-3-formylphenothiazine $(0.037 \mathrm{~g}), 0.17 \mathrm{mmol}$ 2-acetyl-naphthaline $(0.03 \mathrm{~g})$ and $\mathrm{NaOH}$ catalyst was subjected to microwave irradiation. The reaction product (yellow powder, m.p. $161{ }^{\circ} \mathrm{C}$ ) was obtained according to procedure i) $0.05 \mathrm{~g}$ reaction product using $1 \mathrm{~g}$ silica gel solid support, or according to procedure ii) $0.06 \mathrm{~g}$ reaction product using $50 \mathrm{~mL}$ ethanol solvent.

NMR (400MHz, $\left.\mathrm{CHCl}_{3}-\mathrm{d}_{1}\right): \boldsymbol{\delta}_{\mathbf{H}}(\mathrm{ppm}): 3.41(\mathrm{~s}, 3 \mathrm{H}), 6.82(\mathrm{~d}, 1 \mathrm{H}), 6.84(\mathrm{~d}, 1 \mathrm{H}), 6.97(\mathrm{~m}, 1 \mathrm{H}), 7.15(\mathrm{~d}$, 1H), $7.19(\mathrm{~m}, 1 \mathrm{H}), 7.45(\mathrm{dd}, 1 \mathrm{H}), 7.49(\mathrm{~d}, 1 \mathrm{H}), 7.56(\mathrm{~m}, 1 \mathrm{H}), 7.60(\mathrm{~m}, 2 \mathrm{H}), 7.70(\mathrm{~d}, 1 \mathrm{H}), 7.90(\mathrm{dd}, 1 \mathrm{H})$ $7.93(\mathrm{dd}, 1 \mathrm{H}), 8.01(\mathrm{dd}, 1 \mathrm{H}) 8.09(\mathrm{dd}, 1 \mathrm{H}), 8.53(\mathrm{dd}, 1 \mathrm{H}) . \boldsymbol{\delta}_{\mathbf{C}}(\mathrm{ppm}): 35.6,114.1,114.4,119.9,122.6$, 123.1, 124.0, 126.3, 127.3, 127.7, 128.5, 129.8, 143.7, 144.8, 147.9, 190.1, 124.5; 126.7; 129.4 (two overlapping peaks); 127.8, 128.3, 132.6 (two overlapping peaks), 135.4; 135.8

\section{2,6-Bis[3-(10-methyl-10H phenothiazin-3-yl)-1-oxopropenyl]-pyridine 10}

A mixture of 2 mmol 10-methyl-3-formylphenothiazine ( $0.48 \mathrm{~g}), 1 \mathrm{mmol}$ 2,6 diacetyl-pyridine $(0.16 \mathrm{~g})$ and $\mathrm{NaOH}$ catalyst was subjected to microwave irradiation. The reaction product (orange powder, m.p. $151{ }^{\circ} \mathrm{C}$ ) was obtained according to procedure i) $0.30 \mathrm{~g}$ reaction product using $2 \mathrm{~g}$ silica gel solid support, or according to procedure iii) $0.42 \mathrm{~g}$ reaction product using $50 \mathrm{~mL}$ ethanol solvent.

NMR (400MHz, $\left.\mathrm{CHCl}_{3}-\mathrm{d}_{1}\right): \boldsymbol{\delta}_{\mathbf{H}}(\mathrm{ppm}): 3.43(\mathrm{~s}, 6 \mathrm{H}), 6.84(\mathrm{~d}, 2 \mathrm{H}), 6.94,(\mathrm{~m}, 4 \mathrm{H}), 7.10,(\mathrm{~d}, 2 \mathrm{H}), 7.19$ (t, $2 \mathrm{H}), 7.54(\mathrm{~s}, 2 \mathrm{H}), 7.58(\mathrm{~d}, 2 \mathrm{H}), 7.94(\mathrm{~d}, 2 \mathrm{H}), 8.06(\mathrm{t}, 1 \mathrm{H}), 8.29(\mathrm{~d}, 2 \mathrm{H}), 8.34(\mathrm{~d}, 2 \mathrm{H}) . \boldsymbol{\delta}_{\mathrm{C}}(\mathrm{ppm}): 35.32$; 
$114.44 ; 114.09 ; 119.6 ; 119.92 ; 122.61 ; 123.99,128.44 ; 126.28 ; 127.68 ; 129.33,129.11,132.63 ;$ $138.45,143.80 ; 144.75 ; 147.86 ; 153.6 ; 187.6$.

\section{3,7-Bis[3-(4-nitrophenyl)-1-oxopropenyl]-10-propyl-10H-phenothiazine 11}

A mixture of $0.18 \mathrm{mmol}$ 10-propyl-3,7-diacethylphenothiazine $(0.06 \mathrm{~g}), 0.39 \mathrm{mmol}$ 4nitrobenzaldehyde $(0.06 \mathrm{~g})$ and $\mathrm{NaOH}$ catalyst was subjected to microwave irradiation. The reaction product (dark red powder, m.p. $157{ }^{\circ} \mathrm{C}$ ) was obtained according to procedure i) $0.07 \mathrm{~g}$ reaction product using $1 \mathrm{~g}$ silica gel solid support, or according to procedure iii) $0.09 \mathrm{~g}$ reaction product using $50 \mathrm{~mL}$ ethanol solvent.

NMR (400MHz, $\left.\mathrm{CHCl}_{3}-\mathrm{d}_{1}\right): \boldsymbol{\delta}_{\mathbf{H}}(\mathrm{ppm}): 1.06(\mathrm{t}, 3 \mathrm{H}), 1.88(\mathrm{~m}, 2 \mathrm{H}), 3.92(\mathrm{t}, 2 \mathrm{H}), 6.91(\mathrm{~d}, 2 \mathrm{H}), 7.58(\mathrm{~d}$, $2 \mathrm{H}), 7.81(\mathrm{~d}, 2 \mathrm{H}), 7.78(\mathrm{~m}, 6 \mathrm{H}), 7.87(\mathrm{dd}, 2 \mathrm{H}), 8.24$, (d, 4H). $\boldsymbol{\delta}_{\mathrm{C}}(\mathrm{ppm}): 11.8 ; 20.6 ; 42.4 ; 116.2 ; 119.8$; $121.2 ; 121.4 ; 127.3 ; ; 127.8 ; 129.2 ; 131.4 ; 141.2 ; 145.4 ; 147.6 ; 148.2 ; 189.6$.

\section{3-Formyl-10H-phenothiazine 12}

A mixture of $5 \mathrm{mmol}$ phenothiazine $(1 \mathrm{~g}), 10 \mathrm{mmol}$ urotropine $(1.4 \mathrm{~g})$ and glacial acetic acid $50 \mathrm{~mL}$ was subjected to microwave irradiation; $0.6 \mathrm{~g}$ reaction product (yellow powder, m.p.192 ${ }^{\circ} \mathrm{C}$ ) was obtained according to procedure iii).

NMR (400MHz, $\left.\mathrm{CHCl}_{3}-\mathrm{d}_{1}\right): \boldsymbol{\delta}_{\mathbf{H}}(\mathrm{ppm}): 6.70(\mathrm{~d}, 1 \mathrm{H}), 6.75(\mathrm{dd}, 1 \mathrm{H}), 6.80(\mathrm{t}, 1 \mathrm{H}), 7.00(\mathrm{t}, 1 \mathrm{H}), 7.35$ (s, $1 \mathrm{H}), 7.50(\mathrm{dd}, 1 \mathrm{H}), 7.90(\mathrm{~d}, 1 \mathrm{H}), 9.64(\mathrm{~s}, 1 \mathrm{H})$.

$\boldsymbol{\delta}_{\mathbf{C}}(\mathrm{ppm}): 113.88,113.93,115.77,116.69,123.07,126.24,127.36,127.83,130.43,139.57,147.12$, $148.38,158.93$.

\section{Acknowledgements}

Romanian Ministry of Education and Research is greatly acknowledged for financial support (CEEX 80-D-11)

\section{References and Notes}

1. Surducan, E.; Surducan, V.; Nagy; G., Filip, S. V., Installation for Treatment in Microwave Field, Romanian Patent, RO 00116514 / 2001

2. Surducan, E.; Surducan, V. The process and device for heating fluids without dielectric losses, Romanian Patent RO116515/ 2001

3. Surducan, E.; Surducan, V.; The thermographic transducer for high power microwave radiation, Romanian Patent RO116506/ 2001.

4. Molock, F.; Boykin, D. W. The synthesis of pyridoquinolines with di-alkylaminopropylamine side chains, J. Heterocycl. Chem., 1983, 20, 681-686.

5. Matias C.; Mahamoud A.; Barbe J.; Pradines B.; Doury J.C. Synthesis and antimalarial activity of new 4,6-dialkoxy and 4,6-bis(alkylthio)pyrido[3,2-g]quinoline derivatives, Heterocycles 1996, 43, 1621-1632.

6. Chevalier J.; Atifi S.; Eyraud A.; Mahamoud A.; Barbe J.; Pages J.M.; New pyridoquinoline derivatives as potential inhibitors of the fluoroquinolone efflux pump in resistant Enterobacter aerogenes strains, J. Med. Chem. 2001, 44, 4023-4026 
7. Moldovan, C.; Cristea, C.; Silberg, I. A.; Mahamoud, A.; Deleanu, C.; Barbe J. A convenient route to 1,4-dihydro-3-cyano-10-methyl-pyrido[3,2-g]-quinoline derivatives as key-intermediates for the synthesis of novel MDR reversal agents., J. Heterocycl. Commun., 2004, 10, 19-24.

8. Moldovan, C.; Cristea, C.; Silberg, I. A.; Mahamoud, A.; Deleanu, C.; Barbe J. Reactions of the 3cyano-10-methyl-Pyrido[3,2-g]quinolin-4-one", Studia Univ. "Babes-Bolyai, Chemia, 2004, XLIX 118-122.

9. Filip, S. V.; Silberg, I. A.; Surducan, E.; Vlassa, M.; Surducan, V. Microwave assisted phenothiazines preparation by thionation of diphenylamines, Synth. Commun. 1998, 28, 337-345.

10. Gaina, L.; Cristea, C.; Silberg, I.A.; Lovasz, T.; Udrea, S. Aryl-substituted phenothiazinyl-enones I. Synthesis and structural NMR assignments, Rev. Roum. 2002, 47, 983-988.

11. Marckwald, W., Über derivate der toluylendiamincarbonsäure; m-amino-o-methylchinolin, Ann. Chem. 1893, 274, 360-366.

12. Jordis U.; Sauter F.; Rudolf M.; Cai G., Synthesen neuer chinolon-chemotherapeutika, 1. Mitt.: Pyridochinoline und pyridophenanthroline als "lin-benzo-nalidixinsäure"-derivate, Monatsch. Chem. 1988, 119, 761-780.

(C) 2007 by MDPI (http://www.mdpi.org). Reproduction is permitted for noncommercial purposes. 